\title{
Eficacia de intervenciones de diferente intensidad para la promoción del rastreo de cáncer colorrectal
}

Effectiveness of different intensity interventions to promote colorectal cancer screening

\section{Objetivos}

Determinar si una intervención que usa recursos electrónicos (EHR) y aumentos escalonados del apoyo ayuda, a mejorar la adherencia al cribado de cáncer colorrectal (CCR).

\section{Diseño, lugar y pacientes}

Ensayo clínico controlado, aleatorizado, simple ciego, con cuatro grupos paralelos realizado en 21 centros médicos de atención primaria. Fueron incluidos 4.675 adultos de 50 a 73 años a quienes no se les había realizado rastreo de CCR.

\section{Intervención}

Las intervenciones fueron cuatro:

1) Atención habitual.

2) Intervención automática: correos electrónicos vinculados con los registros de salud.

3) Intervención asistida: intervención automática más asistencia telefónica.
4) Intervención con ayuda personalizada o intervención navegada: intervención asistida más la ayuda de una enfermera entrenada.

Las intervenciones fueron repetidas al siguiente año.

\section{Medición de resultados principales}

El resultado principal fue la proporción de participantes que había realizado al menos una intervención de rastreo de CCR en ambos años definida como: 1) al menos una colonoscopía o sigmoidoscopía; 2) al menos sangre oculta en materia fecal (SOMF) durante en el primer año y SOMF, colonoscopía o sigmoidoscopía durante el segundo.

\section{Resultados}

Los resultados principales se describen en la tabla 1. A mayor intensidad de la intervención se observó una mayor proporción de individuos que realizaron cribado de CCR, fundamentalmente a costa de la prueba de SOMF y con una clara tendencia de tipo "dosis-respuesta".

Tabla 1: proporción de individuos que adhirieron a la recomendación de cribado de cáncer colorrectal por rama.

\begin{tabular}{|c|c|c|c|c|c|}
\hline & & \multirow[b]{2}{*}{ Atención habitual } & \multicolumn{3}{|c|}{ Intensidad de la intervención } \\
\hline & & & Automatizada & Asistida & $\begin{array}{l}\text { Con atención } \\
\text { personalizada }\end{array}$ \\
\hline \multirow{2}{*}{$\begin{array}{l}\text { Proporción de los pacientes que } \\
\text { habían realizado al menos una } \\
\text { intervención de rastreo de CCR ambos } \\
\text { años (IC95\%) }\end{array}$} & $\begin{array}{l}\text { Con algún } \\
\text { método }\end{array}$ & $\begin{array}{c}26,3 \% \\
(23,4 \text { a } 29,2)\end{array}$ & $\begin{array}{c}50,8 \% \\
(47,3 \text { a } 54,4)\end{array}$ & $\begin{array}{c}57,5 \% \\
(54,5 \text { a } 60,6)\end{array}$ & $\begin{array}{c}64,7 \% \\
(62,5 \text { a } 67,0)\end{array}$ \\
\hline & Con SOMF & $\begin{array}{c}3,9 \% \\
(2,8 \text { a } 5,1)\end{array}$ & $\begin{array}{c}27,5 \% \\
(24,9 \text { a } 30)\end{array}$ & $\begin{array}{c}30,5 \% \\
(27,9 \text { a } 33 ; 2)\end{array}$ & $\begin{array}{c}35,8 \% \\
(33,1 \text { a } 38,6)\end{array}$ \\
\hline
\end{tabular}

CCR: cáncer colorrectal. SOMF: sangre oculta en la materia fecal.

\section{Conclusión}

En comparación con la atención habitual, una intervención que usa recursos electrónicos y aumentos escalonados de su intensidad, duplicó la proporción de cribado CCR.
Fuente de financiamiento: Instituto Nacional del Cáncer e Institutos Nacionales de Salud de EE.UU. Conflicto de intereses: no referidos.

\section{Comentario}

Si bien los resultados del estudio que hemos resumido son alentadores, no hay que olvidar que este tipo de investigación puede sobre-estimar la eficacia de este tipo de intervenciones ya que los contextos "controlados" de los ensayos clínicos suelen ser más cercanos a un mundo ideal (eficacia) que las condiciones reales de la práctica de los Centros de Atención Primaria, que es donde transcurre la atención de nuestros pacientes (efectividad) ${ }^{1}$.

Sin embargo el efecto dosis respuesta observado, hace pensar que este tipo de intervención tiene al menos, cierta eficacia. Por otro lado, vale destacar que el mayor aumento incremental de la proporción de personas cribadas atribuible a la intervención, está vinculado a la intervención automatizada a través de mensajes de correo electrónico -la menos onerosa en recursos humanos-, mientras que el aumento incremental asociado a las intervenciones más intensivas es francamente menor.

\section{Conclusiones del comentador}

El rápido crecimiento de los registros electrónicos automatizados proporciona oportunidades para la difusión los dispositivos de apoyo para los prestadores de cuidados de salud. Parece razonable tener en cuenta este tipo de herramientas de recordatorio para contribuir a la adherencia del rastreo de CCR, a lo que podría sumarse el envío postal de "kits" para realizar SOMF a las personas que acepten realizarlo.

Dalia Lewitan [ Servicio de Clínica Médica del Hospital Juan Fernández. dalialewitan@hotmail.com ]

Lewitan D. Eficacia de intervenciones de diferente intensidad para la promoción del rastreo de cáncer colorrectal. Evid Act Práct Ambul. 2014, 17(2). Abr-Jun. 48. Comentado de: Green B y col. An Automated Intervention With Stepped Increases in Support to Increase Uptake of Colorectal Cancer Screening. A Randomized Trial. Ann Intern Med. 2013;158:301-311. PMID: 23460053.

Referencias

1. Conde J. Eficacia y efectividad: una distinción útil para la práctica y la investigación clínicas. Nefrología. Vol. XXII. Número 3. 2002 